regards the accuracy of the construction of these chronographs, the best criterion is to be found in the force that is required to work them. We find that 7 foot-pounds per hour drives the clock-work and pendulum; 7 footpounds more drives the carriage as well; and only 3 footpounds more is wanted for the cylinder-- 17 foot-pounds per hour for the whole instrument. Considering the resistance of the carriage, the resistance of the glycerine, and the weight of the cylinder, we think the result as surprising as it is satisfactory.

\section{THE BELGIAN ENTOMOLOGICAL SOCIETY}

$\mathrm{T}$ N April, 1855, a circular with ten signatures was addressed to entomologists residing in Belgium, proposing the formation of a National Entomological Society, the students of Insecta and allies having at that time no organisation, no central meeting-place for interchange of ideas, no special medium in which to publish the results of their researches. The proposal was met by cordial approval, and the first volume of the Annales of the newly-formed Society, published in $1 \$ 57$, indicated a strength of forty-seven effective and four honorary members, with Baron de Selys-Longchamps as president. At first its publications were occupied almost entirely by subjects concerning the Belgian fauna, the volumes were thin, and each represented the work of more than one year. The Society was however well grounded, and notwithstanding occasional short periods of depression, it gradually in creased in the number of its members, in the wideness of the scope of the papers read at its meetings, and in reputation as one of the leadirg entomological societies. Naturally the size of the volumes of the Annales, and the frequency of their appearance, also increased, and now the Society produces a volume each year that no similar society need be ashamed of. The twenty-second volume appeared in 1879 , showing that the wealness inherent on infancy was soon overcome. The list in this volume shows a total of I7 I effective Members (including many foreigners, of whom, however, only six are our own countrymen), twelve Honorary Members (including Messrs. Stainton and Westwood), with the addition of Corresponding and Associate Members. It had also at that time acquired the distinction of being recognised by the State and of receiving a certain amount of State aid.

On October 18, i880, the Society celebrated its twentyfifth anniversary, rejoicing in the attainment of more than its majority, on which occasion the present President (M. Weinmann) read a short congratulatory address, and the indefatigable secretary (M. Preudhomme de Borre, to whom the Society owes much of its recent success) gave an instructive sketch of the history and progress of the Society. On that occasion an especial honour was bestowed upon its first president, Baron de Selys-Longchamps (recently elected President of the Belgian Senate) by conferring upon him (in spite of his protestations) the title of Honorary President, a graceful tribute to one who for so long had continually used his energies, his social and scientific position, and his purse in furthering its interests.

Even apart from purely scientific considerations, the history of Belgium is so indissolubly mixed up with our own, and the feeling of fraternity so close, that all students of entomology in this country cannot but reciprocate the mutual congratulations that passed on this occasion between the native members; and the hearty and unaffected demonstrations of friendship accorded to our own entomologists who have attended the meetings of the Society show how warmly they welcome those of the foreign members who occasionally visit Brussels. The meetings are held in a room in the Royal Natural History Museum, in which is the library, and in connection with the entomological collection of the Museum. The annual subscription is small, and entitles the members to receive all the publications, including elaborate separate reports of the proceedings at the montbly meetings. We hope soon to see Englishmen figuring far more numerously in the lists of members.

R. MCL.

\section{A GENERAL THEOREM IN KINEMATICS}

$T H E$ following theorem with regard to the motion of a rigid body will doubtless be interesting to mathematical physicists :-

In all cases of the motion, parallel to one plane, of a rigid body there is at every instant a point, $J$, of no acceleration, in the plane of motion; the acceleration of every point, $P$, in the plane is in magnitude directly proportional to the distance, $J P$; and its direction makes with $J P$ an angle which, though varying with the time, is at any instant the same for all points in the body.

If $\omega$ is the angular velocity of the body at any instant and $\dot{\omega}$ the angular acceleration, the angle between $\int \boldsymbol{P}$ and the direction of acceleration of $P$ is

$$
\tan ^{-\mathrm{r}} \frac{\dot{\omega}}{\omega^{20}}
$$

We have therefore in all cases of uniplanar motion of a solid body an instantaneous acceleration centre, which is analogous to the ordinary instantaneous [velocity] centre.

Of course the ordinary equation

$$
I \dot{\omega}=L,
$$

which holds for motion round an axis fixed in space and in the body, and which expresses that the moment of the external forces about the axis is equal to the moment of the forces of inertia, holds also for the instantaneous acceleration centre.

As a particular case, whenever a solid moves with constant angular velocity, the accelerations are all directed towards the same point at any instant, and it follows that the resultant stress exerted over the surface of any little lump of the matter is a force directed to this point, if no continuous forces act.

This centre can be in any case geometrically constructed by a rule analogous to that for the ordinary instantaneous centre.

When I had hit upon this theorem I mentioned it to Prof. Wolstenholme, who at once looked out for its exterision to three dimensional motion. The result is very simple.

In all cases of the motion of a solid body there is at every instant a point, $J$, of no acceleration; and if at $J$ there be drawn two axes, $J A$ and $J B$, which are those respectively round which the resultant angular velocity, $\omega$, and the resultant angular acceleration, $\dot{\omega}$, take place, the acceleration of any point, $P$, is compounded of twoone along the perpendicular, $p$, from $P$ on $J A$, and the other perpendicular to $J B$ and to the perpendicular, $q$, from $P$ on $J B$, these two components being, respectively,

$$
\omega^{2} p \text { and } \dot{\omega} q \text {. }
$$

It seems surprising that such a simple and general property of the motion of a rigid body should not have been well known long ago.

Royal Indian Engineering College,

Cooper's Hill, November 6

\section{NOTES}

THE awards of medals for the present year made by the President and Council of the Royal Society are as follows :-The Copl y Medal to Prof. James Joseph Sylvester, F.K.S., for his long-continued investigations and discoveries in mathematics; a Royal Medal to Prof. Joseph Lister, F.R.S., for his contributions on various physiological and biological subjects published in the Philosophical Transastions and Procedings of the Royal 